\title{
Prevalencia de anquiloglosia en neonatos y relación con datos auxológicos del recién nacido o con otras malformaciones o enfermedades asociadas
}

\author{
Francisco Guinot Jimeno, ${ }^{1}$ \\ Natalia Carranza Bagé, ${ }^{2}$ \\ Ana Veloso Durán, ${ }^{3}$ \\ Silvia Parri Bonet, ${ }^{4}$ \\ Mercè Virolés Suñer. ${ }^{5}$
}

\section{Resumen}

Objetivo: determinar la prevalencia de anquiloglosia en neonatos del Hospital Nostra Senyora de Meritxell del Principado de Andorra, Europa, así como la relación existente con datos auxológicos $\mathrm{u}$ otras malformaciones o enfermedades asociadas. Material y métodos: Se realizó un estudio descriptivo, transversal, retrospectivo poblacional de los recién nacidos durante un período de 8 meses. Se estudiaron once variables: presencia o no de anquiloglosia, tipo de frenillo corto, talla, peso, perímetro craneal, sexo, Rh y grupo sanguíneo, edad gestacional, malformaciones y patologías neonatalesasociadas, deforma confidencial y anónima, correlacionándolas para ver si existía alguna asociación entre ellas. Resultados: Un total de 306 recién nacidos fueron incluidos en el estudio (52\% varones y $48 \%$ mujeres). La prevalencia de anquiloglosia fue del 6,54\% ( $\mathrm{n}=20)$. Del total de varones (159/306), el 8,125\% $(\mathrm{n}=13)$ presentaban anquiloglosia, mientras que en mujeres (147/306), la prevalencia fue del $4,79 \%(\mathrm{n}=7)$, sin encontrar diferencias estadísticamente significativas respecto al sexo ( $\mathrm{p}$-valor $=0,24)$. Según la clasificación de Coryllos, el tipo II fue el más frecuente (95\% de los casos) y el $4,58 \%$ de los lactantes con anquiloglosia exhibieron patología/malformación. Conclusiones: La prevalencia de anquiloglosia en recién nacidos en Andorra, Europa, es similar a la observada por otros autores en investigaciones similares. No se observó asociación entre la presencia o no anquiloglosia y las variables estudiadas.

Palabras clave: Anquiloglosia, lengua atada, neonatos, problemas succión, lactancia materna, frenotomía.

\footnotetext{
${ }^{1}$ Doctor en Odontología. Jefe del Departamento de Odontopediatría de la Universitat Internacional de Catalunya (UIC).

${ }^{2}$ Licenciada en Odontología. Alumna del Máster en Odontopediatría Integral y Hospitalaria de la Universitat Internacional de Catalunya (UIC). ${ }^{3}$ Doctora en Odontología. Profesora Asociada del Departamento de Odontopediatría de la Universitat Internacional de Catalunya (UIC).

${ }^{4}$ Instructura Clínica del Departamento de Odontopediatria de la Universitat Internacional de Catalunya (UIC).

${ }^{5}$ Máster en Odontopediatría. Coordinadora del Máster en Odontopediatría Integral y Hospitalaria de la Universitat Internacional de Catalunya (UIC).
} 
Artigo original

\section{Prevalência de anquiloglossia em recém-nascidos e relação com dados auxológicos do recém-nascido ou com outras malformações ou doenças associadas}

\section{Resumo}

Objetivo: determinar a prevalência de anquiloglossia em recém-nascidos do Hospital Nostra Senyora de Meritxell do Principado de Andorra, Europa, bem como a relação existente com dados auxológicos ou outras malformações ou doenças associadas. Material e métodos: Foi realizado um estudo descritivo, transversal e retrospectivo da população de recém-nascidos durante um período de 8 meses. Onze variáveis foram estudadas: presença ou não de anquiloglossia, tipo de frênulo curto, altura, peso, perímetro craniano, sexo, Rh e grupo sanguíneo, idade gestacional, malformações e patologias neonatais associadas, de forma confidencial e anônima, correlacionando-as para ver se existia alguma associação entre eles. Resultados: Um total de 306 recémnascidos foram incluídos no estudo (52\% masculino e $48 \%$ feminino). A prevalência de anquiloglossia foi de $6,54 \%(n=20)$. Do total de homens (159/306), 8.125\% ( $n=13)$ apresentavam anquiloglossia, enquanto nas mulheres (147/306) a prevalência foi de $4,79 \%(n=7)$, sem encontrar diferenças estatisticamente significantes em relação ao sexo (valor-p =0,24). De acordo com a classificação de Coryllos, o tipo II foi o mais frequente (95\% dos casos) e 4,58\% dos lactente com anquiloglossia apresentaram patologia / malformação. Conclusões: A prevalência de anquiloglossia em recém-nascidos em Andorra, Europa, é semelhante à observada por outros autores em investigações semelhantes. Não foi observada associação entre a presença ou não anquiloglossia e as variáveis estudadas.

Palavras-chave: Anquiloglossia, língua amarrada, neonatos, problemas de sucção, amamentação, frenotomia.

Original article

\section{Prevalence of ankyloglossia in neonates and relationship with auxological data of the newborn or with other malformations or associated diseases}

\begin{abstract}
Aim: to determine the prevalence of ankyloglossia in new-borns of the Nostra Senyora de Meritxell Hospital in the Principado de Andorra, Europe, as
\end{abstract}

well as the existing relationship with auxological data or other malformations or associated diseases. Material and methods: A descriptive, cross-sectional, retrospective population study of 306 newborns was carried out over a period 
of 8 months. Eleven variables were studied: presence or no of ankyloglossia, type of short frenulum, height, weight, cranial perimeter, sex, Rh and blood group, gestational age, malformations and associated neonatal pathologies, confidentially and anonymously, correlating them to see if there was any association between them. Results: A total of 306 new-borns were included in the study (52\% male and $48 \%$ female). The prevalence of ankyloglossia was 6,54\% $(n=20)$. Of the total of men (159/306), $8,125 \%(n=13)$ presented ankyloglossia, while woman (147/306), the prevalence was $4,79 \% \quad(n=7)$, without finding

\section{Introducción}

La prevalencia de anquiloglosia (AG) en neonatos es variable en la literatura por la falta de definición uniforme al realizar su diagnóstico. ${ }^{1}$ Esta anomalía puede contribuir a dificultar la lactancia materna, por lo que es importante estudiar factores que puedan estar relacionados. ${ }^{2,3}$

El frenillo lingual es una mucosa de tejido blando que se extiende desde la superficie ventral de la lengua, en la línea media, hasta el suelo de la boca, asegurando los movimientos de la lengua. ${ }^{2} \mathrm{La}$ lengua atada o AG se describe como una anomalía oral congénita caracterizada por un frenillo lingual anormalmente corto con fijación anterior cerca de la punta de la lengua. Esto produce una adhesión de la lengua al suelo de la boca dando como resultado trastornos del habla, anomalías ortodóncicas, dificultades con la lactancia, falta de crecimiento, daño en el pezón materno/senos, suministro deficiente de leche, ingurgitación mamaria y rechazo a la lactancia materna. ${ }^{2-8}$ statistically significant differences regarding sex ( $p$-value $=0,24)$. According to the Coryllos classification, type II was the most frequent (95\% of cases) and 4,58\% of infants with ankyloglossia exhibited pathology/malformation. Conclusions: The prevalence of ankyloglossia in newborns in Andorra, Europe, is similar to that observed by other authors in similar research. No association was observed between the presence or not of ankyloglossia and the variables studied.

Key words: Ankyloglossia, tongue tie, neonates, sucking problems, breast feeding, frenotomy.

La prevalencia de AG en lactantes va del $0,1 \%$ al $10,7 \%$ y es más común en el sexo masculino en una proporción de 1,5 a 2,6:1 respecto a las niñas, con altas correlaciones familiares. ${ }^{3,5-7,9,10}$ En la mayoría de los casos, la AG se considera un hallazgo aislado en niños; sin embargo, existe evidencia de que los componentes genéticos podrían estar involucrados en la herencia y manifestación en la lengua. ${ }^{11}$

La lengua juega un papel muy importante en la lactancia materna. Para quela función de succión ocurra de forma natural se requiere una coordinación de los procesos de succión, deglución y respiración; en todos estos sucesos interviene de manera activa la lengua, siendo necesaria una adecuada motricidad. ${ }^{12,13}$ Así pues, la existencia de un frenillo lingual corto en el recién nacido puede dar lugar a dificultades para amamantar, como el cierre ineficaz, la transferencia inadecuada de leche y el dolor en el pezón materno. Los problemas se manifiestan rápidamente después del nacimiento. Las dificultades de cierre aparecen durante las primeras 
24 horas y el dolor en el pezón al segundo día. ${ }^{13} \mathrm{La}$ asociación entre la dificultad de amamantary AG fue reportada ya hace más de 500 años. Para una correcta lactancia materna, los movimientos de protrusión lingual y peristalsis son necesarios. En pacientes con frenillo lingual corto, no es posible realizar la peristalsis por lo que hay dificultades en el amamantamiento.

Actualmente, debido a que la lactancia materna es ampliamente reconocida como el método de alimentación óptimo para neonatos, la justificación de la frenotomía suma una nueva finalidad además de mejorar los problemas del habla: facilitar la lactancia materna. ${ }^{14,15}$

Hoy en día los criterios clínicos para diagnosticar la AG varían significativamente en la literatura. Diagnosticar alteraciones del frenillo requiere un conocimiento profundo del evaluador sobre la anatomía de la lengua y los diferentes aspectos del frenillo y las regiones adyacentes. ${ }^{16}$ Para diagnosticar la AG es necesario la presencia de un signo (frenillo corto) y un síntoma (dificultad fonatoria, problemas ortodónticos, etc.).
La evaluación en recién nacidos generalmente comprende la observación visual de la existencia o no del frenillo corto, realizando un examen intraoral completo. La inspección de la lengua, palpación y su función deben formar parte de la primera visita odontológica. El odontopediatra debe examinar el aspecto de la lengua, la existencia o no de lateralización, la elevación, la extensión y expansión de la parte anterior de la lengua cuando el bebé llora o trata de extender la lengua. ${ }^{17}$

Las clasificaciones del frenillo lingual se utilizan para evaluar y caracterizar la estructura en condiciones normales y alteradas. Entre las más aceptadas se encuentra la herramienta de evaluación Hazelbaker (HATLFF), que fue diseñada para evaluar el amamantamiento en los recién nacidos con AG y su severidad. ${ }^{10}$ Por otro lado, la escala LACH evalúa la eficacia de la lactancia materna e incluye en su evaluación a la madre y a su hijo. ${ }^{12,18}$ Una de las clasificaciones anatómicas del frenillo lingual es la de Coryllos, que clasifica el frenillo lingual dependiendo de la cercanía de éste del ápice lingual, basándose en 4 tipos $^{19}$ (Figura 1).

\begin{tabular}{|l|l|l|l|}
\hline Tipo I & Tipo II & Tipo III & Tipo IV \\
\hline $\begin{array}{l}\text { Frenillo fino y } \\
\text { elástico; la lengua } \\
\text { está anclada } \\
\text { desde la punta } \\
\text { hasta el surco } \\
\text { alveolar y se } \\
\text { observa en forma } \\
\text { de corazón. }\end{array}$ & $\begin{array}{l}\text { Frenillo fino y } \\
\text { elástico; la lengua } \\
\text { está anclada } \\
\text { desde 2-4 mm } \\
\text { de punta hasta } \\
\text { el cerca surco } \\
\text { alveolar. }\end{array}$ & $\begin{array}{l}\text { Frenillo grueso } \\
\text { y fibroso y no } \\
\text { elástico; la lengua } \\
\text { está anclada } \\
\text { desde la mitad de } \\
\text { la lengua hasta el } \\
\text { suelo de la boca. }\end{array}$ & $\begin{array}{l}\text { El frenillo no se } \\
\text { ve, se palpa, con } \\
\text { un anclaje fibroso } \\
\text { o submucoso } \\
\text { grueso y brillante } \\
\text { desde la base de } \\
\text { la lengua hasta el } \\
\text { suelo de la boca. }\end{array}$ \\
\hline
\end{tabular}

Figura 1. Clasificación anatómica del frenillo lingual utilizada en el presente estudio de investigación. 
El diagnóstico y manejo de la lengua atada sigue siendo controvertido. Teniendo en cuenta el beneficio que aporta la lactancia materna para el recién nacido, es importante que el profesional sanitario aborde cualquier condición que pueda perjudicarla ${ }^{20}$; cobra importancia su detección precoz realizando un análisis exhaustivo para prevenir dificultades de la alimentación.

Por ello, el objetivo de este estudio fue determinar la prevalencia de AG en recién nacidos del Hospital Nostra Senyora de Meritxell del Principado de Andorra, Europa, así como la relación existente con datos auxológicos $\mathrm{u}$ otras malformaciones o enfermedades asociadas.

\section{Material y métodos}

\section{Diseño del estudio y población:}

Se realizó un estudio descriptivo, transversal, retrospectivo poblacional de los recién nacidos en el Hospital Nostra Senyora de Meritxell de Andorra, Europa, durante un período de 8 meses.

\section{Criterios de inclusión y exclusión:}

Los criterios de inclusión fueron: pacientes nacidos durante el periodo julio 2018 a febrero 2019 (ambos meses inclusive) y de ambos sexos. Los criterios de exclusión fueron: bebés que hubieran nacido en otros centros médicos, aunque tras 24 horas acudieran al Hospital Nostra Senyora de Meritxell de Andorra, los trasladados por urgencia a centros de otros países tras nacer, así como también los que presentaban historias clínicas con falta de información.
Procedimiento y análisis estadístico:

El estudio se evaluó y aprobó por el Comité Ético de Investigación Clínica (CEIC) del Hospital Nostra Senyora de Meritxell en 2019 y posteriormente, por el Comité ético de Investigación (CER) de la Universitat Internacional de Catalunya (UIC), Barcelona, España (TFG-2018/201924).

Se realizó la recogida de información de las historias clínicas de los pacientes, de forma confidencial y anónima, registrándose en una base de datos para finalmente correlacionarlos y comprobar la existencia, o no, de alguna asociación con los parámetros estudiados.

Las variables recogidas para el estudio fueron las siguientes: presencia o no de anquiloglosia, tipo de frenillo según la clasificación de Coryllos $^{19}$ (Figura 1), talla, peso, perímetro craneal (PC), sexo, $\mathrm{Rh}$ y grupo sanguíneo, malformaciones y patologías neonatales asociadas y edad gestacional.

Para la tabulación y análisis de los datos se utilizó el programa estadístico Statgraphics ${ }^{\circledR}$ Centurion XVI Versión 16.0.07. Los resultados de las evaluaciones se presentaron en forma de distribución de la frecuencia y porcentaje de las variables estudiadas.

Las variables categóricas se analizaron mediante la prueba Chi Cuadrado y las numéricas con la prueba de la t de Student. En el momento que la distribución de los puntajes de alguno de los grupos no era normal, se empleó la prueba W de Mann Whitney. 
Se consideró estadísticamente significativo un $\mathrm{p}$-valor $\leq 0,05$ y que existía tendencia estadísticamente significativa si el p-valor estaba entre 0,05 y 0,1 .

\section{Resultados}

Un total de 306 recién nacidos (RN) en el Hospital Nostra Senyora de Meritxell de Andorra, Europa, fueron incluidos en el estudio ( $52 \%$ varones y $48 \%$ mujeres). El $6,54 \%$ de la muestra $(n=20)$ fue diagnosticada con anquiloglosia.

En relación con la caracterización de la muestra, 160 (52,29\%) de los R.N. eran del sexo masculino, de los cuales el 8,125\% $(\mathrm{n}=13)$ presentaron AG. Así mismo, 146 bebés $(47,71 \%)$ eran del sexo femenino, de los cuales el 4,79\% ( $\mathrm{n}=7)$ presentaron AG. No se encontraron diferencias estadísticamente significativas en relación con el sexo $(p$-valor $=0,24)$.

Según la clasificación anatómica de Coryllos, la forma más frecuente fue el tipo II, presente en el $95 \%$ de los casos $(n=19)$, seguido del tipo I hallado en 1 recién nacido (5\%). Los tipos III y IV no fueron observados en la muestra estudiada.

La distribución según la presencia o no de AG y patología/malformación neonatal, edad gestacional (días), peso al nacer (gr.), grupo sanguíneo $\mathrm{ABO}$ y $\mathrm{RH}$, talla $(\mathrm{cm}) \mathrm{y}$ Perímetro Craneal (PC) $(\mathrm{cm})$ se encuentran descritas en la tabla 1.

El $23,5 \%$ de la muestra estudiada

Tabla 1: Distribución según la presencia o no de anquiloglosia y patología/malformación neonatal, edad gestacional (días), grupo sanguíneo ABO y RH, talla (cm), Perimetro Craneal (PC) (cm) y peso al nacer ( $\mathrm{gr}$ ).

\begin{tabular}{|l|c|c|c|}
\hline Variables & $\begin{array}{c}\text { No Frenillo Corto } \\
\mathrm{n}(\%)\end{array}$ & $\begin{array}{c}\text { Si Frenillo Corto } \\
\mathrm{n}(\%)\end{array}$ & p-valor \\
\hline Patología/malformación neonatal & & & \\
\hline No patología/malformación & $220(76,90)$ & $14(70)$ & 0,48 \\
\hline Si patología/malformación & $66(23,10)$ & $6(30)$ & \\
\hline Edad gestacional $(\overline{\mathrm{X}}$ días $)$ & 273,294 & 274,6 & 0,67 \\
\hline Grupo sanguíneo y Rh & & & \\
\hline Grupo sanguíneo A & $140(49)$ & $12(60)$ & 0,65 \\
\hline Grupo sanguíneo B & $24(8,40)$ & $1(5 \%)$ & \\
\hline Grupo sanguíneo O & $110(38,40)$ & $7(35)$ & \\
\hline Grupo sanguíneo AB & $12(4,20)$ & - & \\
\hline Rh+ & $239(84)$ & $15(75)$ & 0,32 \\
\hline Rh- & $47(16,44)$ & $5(25)$ & \\
\hline Talla $(\bar{X}$ cm) & 48,78 & 49,1 & 0,87 \\
\hline Parámetro craneal $(\bar{X} \mathrm{~cm})$ & 34,1028 & 34,1 & 0,84 \\
\hline Peso $(\overline{\mathrm{X}}$ g) & 3092,86 & 3131,5 & 0,49 \\
\hline
\end{tabular}


( $\mathrm{n}=72)$ presentaba alguna patología/ malformación neonatal; de éstos, 6 recién nacidos fueron diagnosticados con frenillo corto, no encontrando diferencias estadísticamente significativas respecto al recién nacido con alguna patología/malformación y frenillo normal ( $\mathrm{p}$-valor $=0,48)$.

El estudio de la edad gestacional al nacimiento tampoco reveló diferencias estadísticamente significativas ( $\mathrm{p}$-valor $=0,67$ ) entre los recién nacidos con anquiloglosia, 274,6 días de gestación de media, frente a los 273,29 días de gestación entre los neonatos sin frenillo corto.

Con relación a la variable grupo sanguíneo $\mathrm{ABO}$ y Rh de toda la muestra estudiada, el grupo mayoritario fue el A $(49,6 \% ; 152 / 306)$ y el Rh más frecuente, el positivo $(83 \%$; 254/306). No se encontraron diferencias significativas entre los diferentes grupos sanguíneos $\mathrm{ABO}(\mathrm{p}=0,65)$, el $\mathrm{Rh}(\mathrm{p}=0,32)$ y el hecho de presentar o no anquiloglosia.

Respecto del estudio auxológico al nacimiento, se valoró el peso, la talla y el perímetro craneal. En ninguno de los tres parámetros estudiados se encontraron diferencias estadísticamente significativas al compararlas con el hecho de presentar o no frenillo corto al nacer: peso $(p=0,49)$, talla $(p=0,87)$, perímetro craneal $(p=0,84)$.

\section{Discusión}

La prevalencia de anquiloglosia en lactantes es variable en la literatura, 21 lo que refleja la falta de una definición coherente; las estimaciones varían del
$4,2 \%$ al $10,7 \%$ en recién nacidos. La prevalencia de anquiloglosia en este grupo de estudio fue del 6,54\%. Este valor es similar al obtenido por otros autores. ${ }^{22,23}$ Sin embargo, González Jiménez et al. ${ }^{24}$ obtuvieron una prevalencia del 12\% de anquiloglosia en la población general de Asturias, un valor que es de 2 a 3 veces mayor que el obtenido por Messner y Lalakea ${ }^{22}$, quienes encontraron una prevalencia del 4,8\% y al de Ricke et al., ${ }^{23}$ con una prevalencia del 4,2\%. Este valor tan alto del estudio asturiano, comparado con lo publicado en otros estudios, se debe a nacidos cuyas familias asistían a un servicio especializado en lactancia materna que se ocupaba de problemas derivados de la misma.

Según lo reportado en la literatura, la anquiloglosia es más común en hombres que en mujeres. Los estudios realizados $5,10,22,25$ en recién nacidos documentan una prevalencia de 1,6 a 3 veces superior en varones que en mujeres. En este estudio también fue observada una ligera diferencia (no estadísticamente significativa) entre mujeres y hombres en los niños con anquiloglosia, a favor de los varones $(8,125 \%$ de los niños y $4,79 \%$ de las niñas). Esto podría ser debido a un sesgo de muestreo de nuestros pacientes, ya que en este estudio $y$, teniendo en cuenta la baja prevalencia de anquiloglosia, la muestra estudiada es pequeña. Así mismo, se cree que la mayoría de los casos de anquiloglosia son esporádicos y tienen una mayor predilección masculina que los casos familiares. ${ }^{26}$ Por ello, se sospecha una etiología genética. ${ }^{9,17,22}$ Sin embargo, es difícil definir la herencia de una condición siendo su diagnóstico meramente retrospectivo. 
Uno de los principales problemas no resueltos de la anquiloglosia es su clasificación y diagnóstico. En este estudio existe una gran controversia debido a la falta de una definición y diagnóstico universalmente aceptada y a la inexistencia de consenso en cuanto a la relación entre la sintomatología presentada por las madres lactantes y el grado de anquiloglosia de los bebés. La clasificación de Coryllos $^{19}$ permite identificar frenillos cortos de tipo III y IV, que pueden pasar desapercibidos a simple vista, mediante palpación. ${ }^{11,24}$ Para el diagnóstico de anquiloglosia en recién nacidos es necesario combinar criterios funcionales y anatómicos..$^{9,24}$

Haham et al. ${ }^{27}$ no encontraron ninguna correlación estadística entre el tipo de frenillo lingual de Coryllos ${ }^{19}$ y la presencia o no de problemas con la lactancia. La herramienta de evaluación Hazelbaker ${ }^{10}$ para la función del frenillo lingual (HATLFF) se ha desarrollado con el fin de valorar cuantitativamente el frenillo lingual y se recomienda para evaluar y justificar la necesidad de realizar una frenotomía. ${ }^{20,28}$

En el presente estudio, los pediatras del Hospital Nostra Senyora de Meritxell de Andorra utilizaron la clasificación de Coryllos como medio y criterio diagnóstico, asociando la limitación de la movilidad lingual con dificultades en la succión y el agarre al pecho. En el estudio de González Jiménez et al. ${ }^{24}$ el tipo II fue el más frecuente (54\%), siguiendo la clasificación de Coryllos. En este estudio también fue el más frecuente, encontrándose en el $95 \%$ de los casos diagnosticados con anquiloglosia.

Uno de los objetivos de la presente investigación era valorar la relación entre presentar anquiloglosia y asociación, o no, con alguna enfermedad o patología. Los resultados sugieren que no hay relación alguna. En la mayoría de los casos, ${ }^{21,29}$ la anquiloglosia se considera un hallazgo aislado en niños, no asociado a otra anomalía o enfermedad congénita. Sin embargo, algunos estudios $5,10,16,29$ observan asociación con el síndrome de paladar hendido ligado a $X$, síndrome de Kindler, síndrome de van der Wounde, y síndrome de Opitz. Por otra parte, otros estudios ${ }^{5,16,29}$ mencionan que la mayoría de los casos son esporádicos, pero las mutaciones en el factor de transcripción TBX22 de T-box pueden causar una anquiloglosia hereditaria. ${ }^{12,16,29}$

En cuanto al resto de variables estudiadas (edad gestacional, peso al nacer, grupo sanguíneo $\mathrm{AB} 0$ y $\mathrm{Rh}$, talla y perímetro craneal) no se encontró ninguna asociación con la presencia o no de anquiloglosia en los neonatos. Holkar RR et al. ${ }^{30}$, tampoco encontraron diferencias estadísticamente significativas entre la anquiloglosia y la dificultad con la lactancia materna y otros parámetros como el sexo, la paridad, el modo de parto o el orden de nacimiento. Es probable que no se haya encontrado evidencia que avale esto ya que el crecimiento intrauterino es un proceso complejo en el que intervienen muchos factores. ${ }^{31}$ La expresión génica correcta de factores de transcripción y de crecimiento tisulares están determinados por la salud del sistema maternofetoplacentario. ${ }^{31}$ Por ello, factores externos deíndole nutricional, patogénico o infeccioso que afecten a este complejo serán determinantes en el desarrollo fetal, tanto funcional como auxológico. Al estar la anquiloglosia relacionada con factores de transcripción y 
no con factores externos como sí lo está la auxología al nacimiento, es lógico pensar que sean variables independientes, sin asociación estadística, como se desprende de nuestro estudio. Respecto al sistema $\mathrm{ABO}$ y Rh, la expresión de sus antígenos sí está determinada genéticamente; sin embargo, no fue hallada ninguna asociación con el hecho de presentar o no anquiloglosia.

\section{Limitaciones de la investigación:}

Se incluyó un número de muestra pequeño ( $n=306$ ) debido a que el índice de natalidad en Andorra fue de 588 nacimientos en el año 2017, tal y como aparece publicado en la Web del Gobierno de Andorra. Sería necesario ampliar el periodo de estudio para poder así obtener un tamaño muestral más amplio y valorar si existen, o no, diferencias estadísticamente significativas.
Podría ser muy interesante poder tener en cuenta el estudio de factores genético/ hereditarios involucrados, y determinar si existe una relación entre la presencia de anquiloglosia y estos factores. Por ello, es necesario seguir estudiando esta área.

\section{Conclusiones}

La prevalencia de anquiloglosia en recién nacidos del Hospital Nostra Senyora de Meritxell del Principado de Andorra fue del $6,54 \%$, valor similar al encontrado por otros investigadores en estudios semejantes.

No se encontraron diferencias relación a la presencia o no de anquiloglosia y el resto de las variables analizadas en el presente estudio: tipo de frenillo según la clasificación de Coryllos, talla, peso, perímetro craneal, sexo, $\mathrm{Rh} \mathrm{y}$ grupo sanguíneo, malformaciones y patologías neonatales asociadas y edad gestacional.

\section{Referencias bibliográficas}

1. Bin-Nun A, Kasirer YM, Mimouni FB. A Dramatic Increase in Tongue Tie-Related Articles: A 67 Years Systematic Review. Breast feed Med. 2017; 12(7): 410-4.

2. Meenakshi S, Jagannathan N. Assessment of lingual frenulum lengths in skeletal malocclusion. J Clin Diagnostic Res. 2014; 8(3): 202-4.

3. Brinkmann S, Reilly S, Meara JG. Management of tongue-tie in children: A survey of paediatric surgeons in Australia. J Paediatr Child Health. 2004; 40(11): 600-5.

4. Jackson R. Improving breastfeeding outcomes: the impact of tongue-tie. Community Pract. 2012; 85(6): $42-4$.

5. Suter VGA, Bornstein MM. Ankyloglossia: Facts and Myths in Diagnosis and Treatment. J Periodontol. 2009; 80(8): 1204-19.

6. Ruffoli R, Giambelluca MA, Scavuzzo MC, Bonfigli D, Cristofani R, Gabriele M, et al. Ankyloglossia: Amorpho functional investigation in children. Oral Dis. 2005; 11(3): 170-4.

7. Block SL. Ankyloglossia: when frenectomy is the right choice. Pediatr Ann. 2012; 41(1): 14-6.

8. Jang SJ, Cha BK, Ngan P, Choi DS, Lee SK, Jang I. Relationship between the lingual frenulum and craniofacial morphology in adults. Am J Orthod Dentofac Orthop. 2011; 139(4): 361-7. 
9. Ferrés-Amat E, Pastor-Vera T, Rodriguez-Alessi P, Mareque-Bueno J, Ferrés-Padró E. The prevalence of ankyloglossia in 302 newborns with breastfeeding problems and sucking difficulties in Barcelona: A descriptive study. Eur J Paediatr Dent. 2017; 18(4): 319-25.

10. Power RF, Murphy JF. Tongue-tie and frenotomy in infants with breastfeeding difficulties: Achieving a balance. Arch Dis Child. 2015; 100(5): 489-94.

11. Coryllos E, Genna CW, Salloum AC. Congenital tongue-tie and its impact on breastfeeding. Am Acad Pediatr. 2004: 1-12.

12. Srinivasan A, Al Khoury A, Puzhko S, Dobrich C, Stern M, Mitnick H, et al. Frenotomy in Infants with Tongue-Tie and Breastfeeding Problems. J Hum Lact. 2018; 1-7.

13. Kupietzky A, Botzer E. Ankyloglossia in the infant and young child: clinical suggestions for diagnosis and management. Pediatr Dent. 2005; 27(1): 40-6.

14. Obladen M. Much ado about nothing: two millennia of controversy on tongue-tie. Neonatology. 2010; 97(2): 83-9.

15. Schafer R, Genna CW. Physiologic Breastfeeding: A Contemporary Approach to Breastfeeding Initiation. J Midwifery Womens Health. 2015; 60(5): 546-53.

16. Huang YS, Quo S, Berkowski AJ, Guilleminault C. Short Lingual Frenulum and Obstructive Sleep Apnea in Children. Int J Pediatr Res. 2015; 1:003.

17. Ricke LA, Baker NJ, Madlon-Kay DJ, DeFor TA. Newborn tongue-tie: prevalence and effect on breastfeeding. J Am Board Fam Pract. 2005; 18(1): 1-7.

18. Kumar SP, Mooney R, Wieser LJ, Havstad S. The LATCH scoring system and prediction of breastfeeding duration. J Hum Lact. 2006; 22(4): 3991-7.

19. Genna CW, Coryllos E V. Breastfeeding and Tongue-Tie. J Hum Lact. 2009; 25(1): 111-2.

20. Edmunds J, Miles SC, Fulbrook P. Tongue-tie and breastfeeding: a review of the literature. Breastfeed Rev. 2011; 19(1): 19-26.

21. Rowan-Legg A. Ankyloglossia and breastfeeding. Paediatr Child Health. 2015; 20(4): 209-13.

22. Messner AH, Lalakea ML. Ankyloglossia: controversies in management. Int J Pediatr Otorhinolaryngol. 2000; 54(2-3): 123-31.

23. Ricke LA, Baker NJ, Madlon-Kay DJ, DeFor TA. Newborn tongue-tie: prevalence and effect on breastfeeding. J Am Board Fam Pract. 2005; 18(1): 1-7.

24. González Jiménez D, Costa Romero M, Riaño Galán I, González Martínez MT, Rodríguez Pando MC, Lobete Prieto C. Prevalencia de anquiloglosia en recién nacidos en el Principado de Asturias. Anales de Pediatría. 2014; 81(2): 115-9.

25. Ballard JL, Auer CE, Khoury JC. Ankyloglossia: assessment, incidence, and effect of frenuloplasty on the breastfeeding dyad. Pediatrics. 2002; 110(5): 63.

26. Walsh J, Tunkel D. Diagnosis and treatment of ankyloglossia in newborns and infants: A review. JAMA Otolaryngol Head Neck Surg. 2017; 143(10): 1032-9.

27. Haham A, Marom R, Mangel L, Botzer E, Dollberg S. Prevalence of Breastfeeding Difficulties in Newborns with a Lingual Frenulum: A Prospective Cohort Series. Breastfeed Med. 2014; 9(9): 438-41.

28. Amir LH, James JP, Donath SM. Reliability of the Hazelbaker assessment tool for lingual frenulum function. Int Breastfeed J. 2006; 1(1): 3.

29. Segal LM, Stephenson R, Dawes M, Feldman P. Prevalence, diagnosis, and treatment of ankyloglossia. Can Fam Physician. 2007; 53(6): 1027-33.

30. Holkar RR, Korday CS, Malik S. Ankyloglossia and its impact on breastfeeding: A prospective observational study. Int J Contemp Pediatr. 2017; 4(4): 1296-301.

31. Paisán Grisolía L, Sota Busselo I, Muga Zurriarían O, Imaz Murgiondo M. El recién nacido de bajo peso. En: Protocolos Diagnóstico Terapticos de la AEP: Neonatología. 2008. Disponible en: www.aeped.es/ protocolos/.

Recibido: $24 / 03 / 2020$

Aceptado: 07/05/2020

Correspondencia: Francisco Guinot Jimeno, correo: fguinot@uic.es 